\title{
Children Dental Trauma Facts for Emergent Field Management
}

Asian Journal of Sports Medicine, Volume 3 (Number 1), March 2012, Page: 64

Oral Trauma constitutes one-fifth of total trauma among children. Dental trauma is the most frequent oral trauma of which tooth avulsion is the most serious injury ${ }^{[1]}$. A common scenario that may be encountered by any football trainer is a child who comes up with a tooth is his hand. A quality and professional approach indeed enhance the prognosis of the avulsed tooth. problem happens?

Consider the front tooth of a 9-year old boy has avulsed due to a direct ball trauma while playing soccer. There are a few steps to perform step by step:

1. Keep calm (both the patient and parents/coach/ paramedics)

2. Ensure that the tooth is permanent

There is a 3-step development of dental structures depending on the type of the teeth: first, deciduous dentition which starts by eruption of the lower incisors (front tooth) at the age of 6-months. Deciduous teeth begin to fall out around the age of 6-7 years. Second, mixed dentition in which, deciduous teeth are replaced by the permanent ones that lasts up to the age of 12 . Third, permanent dentition that comprises permanent teeth with the wisdom teeth and last appears in the oral cavity around the age of 18-22 years ${ }^{[2]}$. Note that deciduous teeth should not be reimplanted in their alveolar socket in the case of traumatic avulsion, since this procedure may damage the underlying permanent tooth bud. For example a front tooth of 9-year old boy is permanent, while the front tooth of a 5-year old child is not.

3. Seek the avulsed tooth. The tooth should be held from the crown (upper part), not from the apex (yellowish bottom of the tooth). If it is dirty, it should be cleaned under cold running tap water (10 seconds). Never brush or clean the tooth with gauze and do not use any disinfectants such as alcohol or povidone iodine. These solutions are toxic for periodontal ligament cells which are crucial to

maintaining the vitality of the avulsed tooth.

4. Consider careful re-implantation of the avulsed tooth. If not possible, the tooth should be kept in a suitable storage medium (e.g., milk, normal saline or child's own saliva). Do not immerse the tooth in alcohol or other disinfectants.

5. Seek an emergent dentist visit. It is proven that the sooner the tooth is re-implanted, the better the prognosis $^{[1]}$.

It seems that dental trauma training is simply overlooked, with more attention paid to more life saving procedures. Any trainer of sport disciplines with high probability of accidental dental traumas should be trained for such limb-saving basic routines when a tooth is avulsed. Moreover, applying mouth guards especially by those with severe class II malocclusion (forward location of upper jaw in relation to the lower jaw with protruded upper front teeth) could considerably reduce the severity of dental trauma. Treatment of a missing tooth due to the previous traumatic avulsion possesses a very heavy psycho-financial burden for both the family and health care services. It is recommended that school sport teachers and professional teachers get licensed for basic routines of dental and more generally orofacial trauma.

Key Words: Oral Trauma; Dental Trauma; Children

\section{Karim Poorsattar Bejeh-Mir, MD ${ }^{1}$; Arash Poorsattar Bejeh-Mir $^{2 *}$}

Authors' Affiliation:

1. Department of Pediatrics, Amir Mazandarani General Hospital, Sari, Iran

2. Dentistry Student Research Committee, Babol University of Medical Sciences, Babol, Iran

* Corresponding Author;

Address: Dentistry Student Research Committee, Babol University of Medical Sciences, Ganj Afrooz Ave., Babol, Iran

E-mail: arashpoorsatrae@yahoo.com

\section{REFERENCES}

1. Flores MT, Andersson L, Andreasen JO, et al. Guidelines for the management of traumatic dental injuries. II. Avulsion of permanent teeth. Dental Traumatol 2007;23:130-6.

2. Johnstone L, Spence D, Kaziol-McClain J. Oral hygiene care in the pediatric intensive care unit: practice recommendations. Pediatr Nurs 2010;36:85-96. 